\title{
Long base-line measurements of short-period mesospheric gravity waves during the SEEK campaign
}

\author{
M.J. Taylor, S.H. Seo \\ Space Dynamics Laboratory and Physics Department, Utah State University, Logan \\ T. Nakamura, T. Tsuda \\ Radio Atmospheric Science Center, Kyoto University, Kyoto, Japan
}

H. Fukunishi, and Y. Takahashi

Department of Geophysics, Tohoku University, Sendai, Japan

\begin{abstract}
Simultaneous observations of short-period ( $<1$ hour) mesospheric gravity waves were made over an extended base-line $(-660 \mathrm{~km})$ from two sites in Japan (at Shigaraki and Yamagawa) during the SEEK (Sporadic-E Experiment over Kyushu) campaign, 9-23 August, 1996. Coincident image data obtained on four nights provided a rare opportunity to investigate the horizontal spatial extent and propagation characteristios of the waves in detail. Surprisingly, out of a total of 12 events observed from Shigaraki and 10 from Yamagawa at most only three exhibited similar propagation characteristics at each site. The majority of the wave displays $(\sim 70-$ 75\%) had quite dissimilar characteristics suggesting a preponderance for localized wave motions of limited lifetimes and geographical extent. A marked preference for wave progression towards the N-NE indicates that the gravity waves imaged in the near infrared $\mathrm{OH}$ and O)(557.7 nm) nightglow emissions were probably not directly associated with the observed E-region irregularities.
\end{abstract}

\section{Introduction}

In recent years sensitive, solid state (CCD) imaging systems have become very powerful, and popular tools for investigating the properties of short-period ( $<1$ hour) gravity waves that propagate through the mesospheric and lower thermospheric (MLT) nightglow layers (height range $\mathbf{- 8 0 - 1 0 0 ~ k m ) . ~ T h e ~ a l l - s k y ~}$ $\left(180^{\circ}\right)$ image format is well suited for these studies as it combines an exceptionally large field of view $\left(-0.75 \times 10^{6} \mathrm{~km}^{2}\right.$ at MLT heights) with good spatial resolution (better than $1 \mathrm{~km}$ in the zenith). Such observations have revealed a wealth of wave structure at mid- and low latitudes characteristic of shortperiod internal gravity waves (IGW) [e.g. Swenson et al., 1995; Taylor et al., 1995; 1997]. A feature of many of these displays is their high degree of spatial and temporal coherence. Often a wave pattern will appear as a succession of several bright "bands" extending from horizon to horizon and encompassing an area of sky much larger than the all-sky field of view (radius $\sim 500 \mathrm{~km}$ ). Figure 1a shows an example of this type of event imaged in the near infrared (NIR) OH emission from Bear Lake Observatory, Utah $\left(41.9^{\circ} \mathrm{N}\right)$. This display lasted for over 4-hrs indicating coherent wave propagation over a very large horizontal distance $(>1000 \mathrm{~km})$ [e.g. Taylor et al., 1995].

Copyright 1998 by the American Geophysical Union.

Paper number $98 \mathrm{GL} 00847$.

0094-8534/98/98GL-00847\$05.00
As most small-scale IGW sources are thought to be localized in nature (resulting from weather related disturbances such as thunderstorms, fronts and jet streams or orographic forcing over mountainous regions) the question arises: how far do shortperiod waves propagate before they dissipate? This information is important for estimating the range over which an individual source can exhibit an influence on the MLT region (by depositing its energy and momentum), but is currently sparse. For freely propagating waves of period $<1$ hour the IGW dispersion relationship indicates a horizontal progression of no more than a few hundred $\mathrm{km}$ from the source region (assuming an isothermal stationary atmosphere) before the waves intercept the MLT region [Hines, 1967]. Observations of short-period waves extending over vast areas therefore suggest that other mechanisms, such as Doppler ducting of the waves at MLT heights (due to strong background wind gradients) can significantly influence the eventual geographic extent and temporal coherence of the wave fields. Indeed, Isler et al. [1997] have shown recently that as many as $\sim 75 \%$ of the short-period wave motions observed in the NIR OH emission (peak height $\sim 87 \mathrm{~km}$ ) and the $\mathrm{OI}(557.7 \mathrm{~nm}$ ) emissions (peak height $-96 \mathrm{~km}$ ) during the ALOHA-93 (Airborne Lidar and Observations of the Hawaiian Airglow) campaign (October 1993) exhibited an evanescent or ducted wave behaviour.

If this condition prevails at other sites and times of the year then we can expect coherent wave progression over very large geographic areas to be a relatively frequent occurrence. However, to date few co-ordinated optical observations have been made to examine the characteristics of short period IGW over an extended area [e.g Swenson et al., 1995]. During the SEEK campaign (9-23 August, 1996) data from two CCD imagers operated in support of sounding rocket measurements [see Fukao et al., this issue] have been used to investigate the occurrence frequencies and properties of short-period waves over an extended base-line $(-660 \mathrm{~km})$. One camera system was located at Yamagawa Observatory in southern Kyushu (in proximity to the launch site at Uchinoura) while the second imager was located at the MU radar site at Shigaraki, Honshu, Japan.

\section{Instrumentation and Observations}

At Yamagawa Observatory $\left(31.2^{\circ} \mathrm{N}, 130.6^{\circ} \mathrm{E}\right)$ a monochromatic CCD imaging system fitted with an all-sky $\left(180^{\circ}\right)$ telecentric lens system and a filter wheel was used to sample sequentially wave structure in the NIR (OH) bands (715-930 


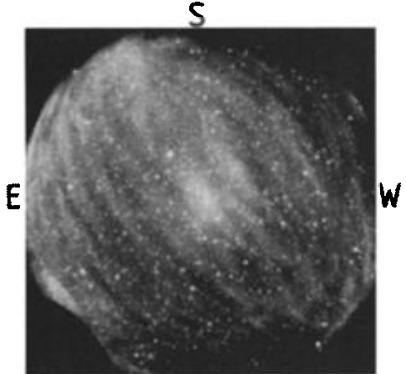

(a)

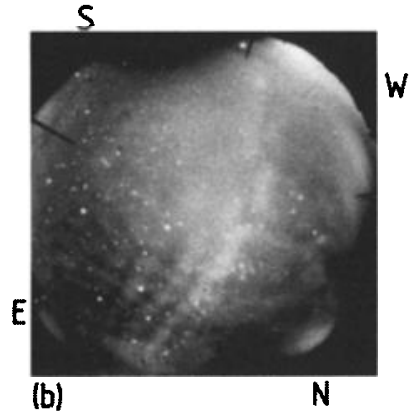

(b)
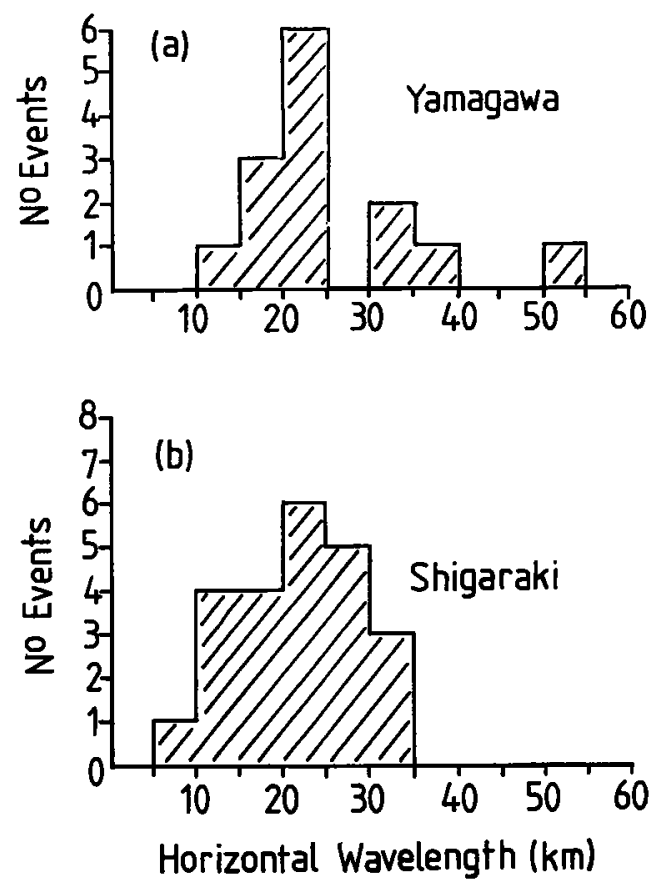

Figure 3. Histogram plots showing the distribution of $\lambda_{\mathrm{l}}$ (binned at $5 \mathrm{~km}$ resclution) for (a) the full Yamagawa $\mathrm{OH}$ and $\mathrm{OI}$ data set and (b) the full Shigaraki OH data set. operated at Shigarari $\left(34.9^{\circ} \mathrm{N}, 136.1^{\circ} \mathrm{E}\right)$ had a somewhat smaller field of view $\left(69^{\circ} \times 52^{\circ}\right)$ and was filtered to observed structure in the broad-band NIR (680-1000 nm) nightglow which is dominated by several $\mathrm{OH}$ Meinel bands [e.g. Swenson et al., 1995]. Data were recorded initially every -4.5 min using an exposure of $120 \mathrm{~s}$, but, after 15 August this was reduced to $\sim 2.5$ min and 60s respectively [see Nakamura et al., this issue].

Figure 2 illustrates the nightglow observing geometry. The circles (all-sky) and the rectangle (narrow angle) indicate the relative fields of view of each imager. The $500 \mathrm{~km}$ radius circle depicts the maximum field of view of the all-sky imager, but, due to limited seeing conditions at Yamagawa most of the wave measurements were made within a $-300 \mathrm{~km}$ radius. As the Shigaraki imager exhibited a relatively small "footprint" at $\mathrm{OH}$ altitudes $(-105 \times 80 \mathrm{~km})$ it was not possible to map wave

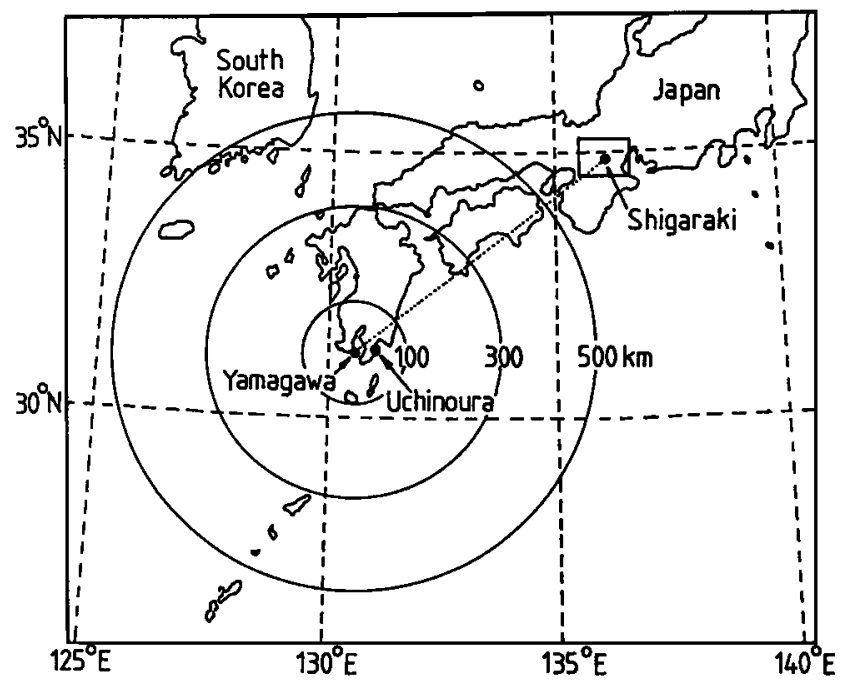

Figure 2. Map showing the location and fields of view of the two imagers. The base-line was $-660 \mathrm{~km}$ at an azimuth of $\sim 50^{\circ}$.

structure directly from one field into the other. Nevertheless, the location of the two sites was such that extensive wave structure present in one image should be identifiable readily in the other.

\section{Results}

Coincident measurements were made during the period 9-23 August, 1996. The observing conditions at Shigaraki were generally good and wave data were recorded on nine nights resulting in a total of $23 \mathrm{OH}$ events [Nakamura et al. 1997, this issue]. In comparison, the seeing conditions at Yamagawa were hampered by high aerosol levels, frequent cloud and the passage of a typhoon. Nevertheless, wave data were imaged successfully on five nights yielding a total of 14 events (each measurement limited to $-0.5-2$ hours duration). Four of these nights (17, 18, 19 and 22 August UT) coincided with the Shigarakj measurements. Figure $1 \mathrm{~b}$ shows an example of the $\mathrm{OH}$ wave data recorded at Yamagawa on 19 August at 15:24 UT. Two spatially limited waves are seen progressing on almost orthogonal headings creating a "cross-batch" pattern (similar waves were evident in the OI emission). These events are representative of many of the displays recorded during the campaign.

Before comparing individual wave events (limited to four night's data) it is instructive to examine the overall characteristics of each data set. Figure 3 shows the histogram distribution of horizontal wavelengths, $\lambda_{1}$, for the full Shigaraki and Yamagawa measurements. Both plots peak over the same wavelength range $(20-25 \mathrm{~km})$ and exhibit comparable mean values. However, a small fraction of the Yamagawa data extend to longer wavelengths that would probably not be evident in the Shigaraki data set due to its limited field of view. The corresponding phase velocity plots (Figure 4) both exhibit marked anisotropy in their azimuthal distributions, with a clear preference for wave propagation towards the $\sim \mathrm{NE}$ and a second maximum towards the $\sim S E$. The Yamagawa data also indicate a 


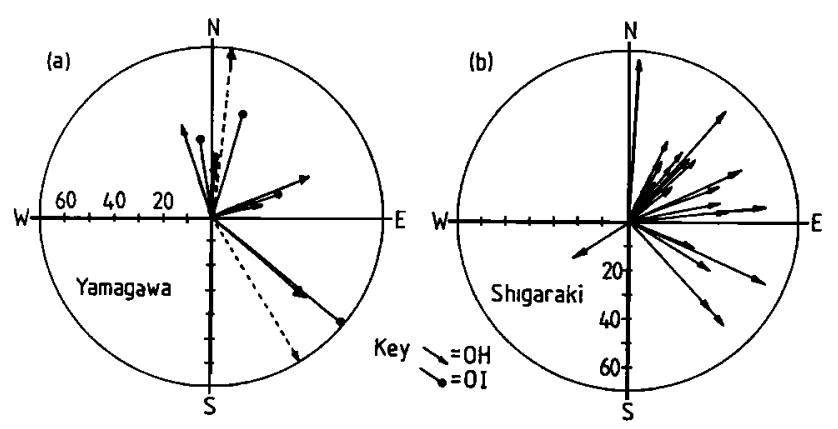

Figure 4. Velocity distributions for (a) the full Yamagawa $\mathrm{OH}$ and $\mathrm{OI}$ data, and (b) the full Shigaraki $\mathrm{OH}$ data. Each vector indicates the magnitude and direction ' $\theta$ ' measured clockwise from $N$. Note the dashed arrows represent events where the wave phase speed was indeterminate.

strong tendency for wave propagation towards the $\mathbf{N}$. Together these results suggest that the waves imaged at both sites during the SEEK campaign were, on average, very similar in their measured propagation characteristics and therefore probably resulted from the same (or similar-type) sources. However, a night-by-night comparison of the wave events suggests a quite different result with very little similarity in either the number of events observed or their measured parameters.

In order to correlate these two data sets it is important to establish first a working criteria for identifying like gravity wave events. For waves generated in the troposphere in the same frame of reference as an observer, both the $\lambda_{2}$ and the observed horizontal phase speed, $v_{h}$, of an event time (and hence its observed period $\tau_{o b}$ ) should remain approximately constant with time. This is because it is the intrinsic period, $\tau_{i}$, of the wave that alters with the varying background wind field, $\overline{\mathrm{U}}$, and is given by the relationship $\tau_{i}=\tau_{o b} \cdot v_{h} /\left(v_{b}-\bar{U}\right)$. The observed direction of motion, $\theta$, however, is dependent on the relative location of the source with respect to the observers. For example, if a quasi-monochromatic source were located between the two sites then each observer would report waves moving in opposite directions (assuming a stationary atmosphere). However, for waves generated by more distant sources the measured direction of the wave fronts should be similar. Thus, our initial approach is to compare all three wave parameters $\left(\lambda_{h}, v_{h}, \theta\right)$ measured at each site to help identify similar wave events.

Table 1 (top) lists the results of the Yamagawa NIR OH and Ol(557.7 nm) measurements for the four coincident data nights (the $\mathrm{Na}$ data have yet to be analysed). For this study waves in the $\mathrm{OH}$ emission were identified first and then supplemented by an analysis of the OI emission for additional events. A total of 10 separate wave displays were recorded. Average values for $\lambda_{\mathrm{b}}$ $=23 \mathrm{~km}, \mathrm{~h}=41 \mathrm{~ms}^{-1}$ and $\tau_{\mathrm{ob}}=10.6 \mathrm{~min}$ are typical for shortperiod gravity waves reported in the literature [e.g. Swenson et al., 1995; Taylor et al., 1995; 1997]. The Shigaraki measurements (total 12 events) obtained on these four nights (Table 1, bottom) indicate almost identical mean values to the Yamagawa data. However, using our selection criteria for identifying like events it is evident that only one display (imaged at 9:15 UT on 22 August from Yamagawa and 18:26 UT at Shigaraki) exhibited similar values for $\lambda_{b}, n$, and $\theta$ (within the experimental uncertainties). This wave was observed to progress towards the $-\mathrm{SE}$ on an almost orthogonal heading to the site base-line. Other events were found that exhibited similarities in one or sometimes two parameters but not all three.

\section{Discussion}

The apparent lack of similarities between these two data sets was unexpected, given the wealth of extensive wave patterns imaged at other sites and times of the year [e.g. Taylor et al., $1995 ; 1997]$. Considering the observing geometry (Figure 2) and the preponderance for wave progression towards the $-\mathrm{NE}$ (i.e. along the site base-line from Yamagawa to Shigaraki), any spatially extensive, long-lastirg, wave pattern (of the type shown in Figure 1a) should have been evident in both data sets. One possible reason for this lack of coincidence is that our selection criteria may be too rigid to account for any evolution of the wave field with time (due to changes in the source region or the background winds). For example, on 22 August the Yamagawa OI wave pattern $\left(\lambda_{b}=33 \mathrm{~km}\right)$ was observed to "split" to form waves of wavelength $\sim 16 \mathrm{~km}$ (results indicated in brackets in Table 1). This phenomena has been reported previously [e.g Herse et al., 1980] and may not be uncommon. Taking this into account suggests two possible additional event pairs. One pair imaged on 17 August (13:40 UT at Yamagawa, 14:54 UT at Shigaraki) which showed clear similarities in phase speed ( 50 $\left.\mathrm{ms}^{-1}\right)$ and direction of motion $\left(\sim 1^{\circ}\right)$ but, significantly different horizontal wavelengths. The other pair were imaged on 18 August (18:22 UT at Yamagawa, 17:43 UT at Shigaraki) and exhibited horizontal wavelengths of 11 and $23 \mathrm{~km}$ suggestive of wavelength "splitting" but no corroborating azimuth or phase speed information are available to support this hypothesis. To

Table 1. Summary of wave events recorded at Yamagawa (top) and Shigaraki (bottom) for the four nights (17, 18, 19 and 22 August UT) of coincident measurements. Note the 17 August UT corresponds to a local date of 17/18 August etc.

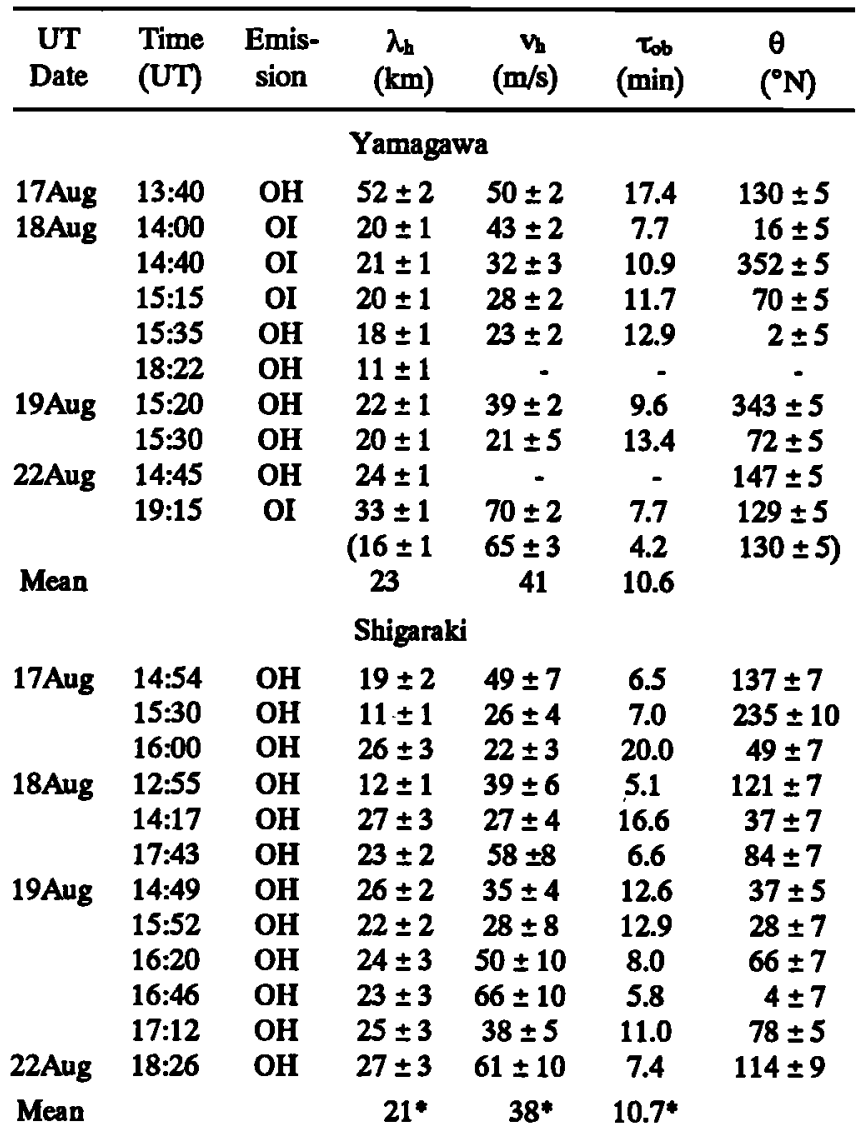

* Note: values for the full OH data set of Nakamura et al. [this issue]. 
account for possible differences in the observed wave headings (due to different source-observer viewing geometries) the criteria for identifying similar values of $\theta$ was also broadened to encompass waves within a $\sim 45^{\circ}$ sector centred on their measured propagation directions but yielded no further events. Thus, by relaxing the selection criteria to account for possible changes in the horizontal wave-field and the propagation direction (but not the observed phase speed) the number of event pairs increases to (at most) three representing only $-25-30 \%$ of the displays observed at each site.

At no time during the SEEK campaign did the all-sky imager detect very extensive wave displays. This result is exemplified in Figure 1b, which shows two superposed wave patterns that were evident only in the lower half of the camera's field. One wave (event a) progressed towards Shigaraki $\left(\theta=72^{\circ}\right)$ while the other (event b) progressed almost normal to the site base-line $(\theta=$ $343^{\circ}$ ). At Shigaraki a total of five events were observed on this night, two of which exhibited similar headings $\left(-70^{\circ}\right)$ to the Yamagawa (event a) wave but their phase speeds were significantly larger (>factor 2). No evidence was found in the Shigaraki data for wave progression towards the $\sim \mathrm{NW}$ in association with event $b$. In summary, the variety of waves imaged during the SEEK campaign suggests that the wave sources were quite numerous but tended to generate limited nightglow signatures comprising only a few wave crests. The apparent absence of extensive, long-enduring displays also suggests little evanescent or ducted behaviour during this period [ls]er et al., 1997].

Apart from tropospheric sources, one potential source mechanism that could generate waves with limited spatial and temporal signatures is in-situ wave generation by strong wind shears (associated with breaking tides and gravity waves). For wave generation by this mechanism the atmosphere has to become unstable requiring a vertical shear in the horizontal winds $(\Delta U)>40 \mathrm{~ms}^{-1} \mathrm{~km}^{-1}$ at MLT heights [e.g. Taylor and Hapgood, 1990]. This mechanism is thought to be responsible for many of the short-lived (several minutes), small-scale "ripple" waves $\left(\lambda_{n} \sim 5-15 \mathrm{~km}\right)$ that are often observed in the nightglow emissions [e.g Taylor and Hapgood, 1990], but, it is not usually considered a source of longer wavelength patterns as significantly larger shears would be needed (as $\lambda_{h} \alpha \Delta U$ ) [e.g. Haurwitz and Fogle, 1969]. However, during the SEEK campaign combined radar and rocket measurements of the neutral winds over southern Japan [see Larsen et al., Figs. 1,4, this issue] have demonstrated the presence of very strong wind shears at -97 and $\sim 103 \mathrm{~km}$, on at least one occasion (20 August), the latter of which exceeded $50 \mathrm{~ms}^{-1} \mathrm{~km}^{-1}$ indicating highly unstable conditions. Such large wind shears are unusual but they have now been observed on several occasions in association with sporadic-E [Larsen et al., this issue]. Unfortunately, no meaningful airglow measurements were obtained from Yamagawa during this night due to clouds. It therefore remains to be seen whether the localized wave events, of $\lambda_{b}-20-30 \mathrm{~km}$ reported here, can be generated by such a mechanism.

\section{Summary}

Contrary to expectations our results indicate a paucity of spatially extensive, coherent waves over central and southern Japan during the SEEK campaign. At most only $25-30 \%$ of the events observed exhibited sufficiently similar wave characteristics $\left(\lambda_{h}, v_{h}, \theta\right)$ to have been measurements of the same display. At no time were extensive "all-sky" wave displays imaged of the type described in Taylor et al., [1995]. Instead, these data suggest a preponderance for localised sources sustaining limited horizontal wave propagation and/or the presence of strong wind filtering or wave dissipation at MLT heights. The origin of these waves is not yet known but given the large prevailing winds in the MLT during this period it is speculated that very strong shears may be a potential source for some of the observed wave structures.

The similar azimuthal distribution for the Yamagawa and Shigaraki data sets indicates wave progression generally towards the $\mathbf{N}$ and $\mathrm{NE}$ which is contrary to the observed motion of quasi-periodic field-aligned irregularities (height $\sim 100-120 \mathrm{~km}$ ) towards the $\sim \mathrm{S}-\mathrm{SE}$. This finding is discussed in more detail by Nakamura et al. [this issue] and indicates that the gravity waves imaged in the $\mathrm{OH}$ and $\mathrm{OI}$ emission (altitude range $\sim 80-100 \mathrm{~km}$ ) were most probably not related to the E-region irregularities.

Acknowledgenents. We are most grateful to the director and staff at Yamagawa Radio Wave Obeervatory for their belp during this campaign. The Utah State University measurements were supported in part by a grant from the Utah Asian Studies Consortium (UCON) and by Kyoto Univessity. The analysis of these data was performed under National Science Foundation grant No ATM-9525815.

\section{References}

Fukao, S., M. Yamamoto, R. Tsunoda, H. Hayakawa, and T. Mukai, The SEEK (Sporadic-E Experiment over Kyushu) campaign, Geophys. Res. Lett., this issue.

Haurwitz, B., and B. Fogle, Wave forms in noctilucent clouds, DeepSea Res., 16, 85-95, 1969.

Herse, M., G. Moreels, and J. Clairemidi, Waves in the OH emissive layer: photogrammetry and topography, Appl. Optics, 19, 355-362, 1980.

Hines, C.O., On the nature of travelling ionospheric disturbances launched by low-altitude nuclear explosions, J. Geophys. Res., 72, 1877-1882, 1967.

Isler, J.R, M.J. Taylor, and D.C. Fritts, Observational evidence of wave ducting and evanescence in the mesosphere, J. Geophys. Res., 102, D22, 26,301-26,313, 1997.

Larsen, M.F., C. Odom, S. Fukao, M. Yamamoto, and K. Igarashi, Observation of unstable neutral wind shears in region of sporadic-E and quasi-periodic plasma structure, Geophys. Res. Lett., this issue.

Nakamura, T., T. Tsuda, H. Miyagawa, Y. Matsushita, H. Fukunishi, Y. Takahashi, and Y. Yamada, Propagation direction of gravity wave pattern observed in $\mathrm{OH}$ CCD image during SEEK campaign, Geophys. Res. Lett., this issue.

Swenson, G.R, M.J. Taylor, P.J. Espy, C. Gardner, and X. Tao, ALOHA. 93 measurements of intrinsic AGW characteristics using airborne airglow imager and groundbased Na Wind/Temperature lidar, Geophys. Res. Lett., 22, 2841-2844, 1995.

Taylor, M.J., and M. A Hapgood, On the origin of ripple-type wave structure in the OH nightglow emission, Planet. Space Sci, 38, 1421$1430,1990$.

Taylor, M.J., M.B. Bishop, and V. Taylor, All-sky measurements of short period waves imaged in the Ol(557.7 $\mathrm{nm}), \mathrm{Na}(589.2 \mathrm{~nm})$ and near infrared $\mathrm{OH}$ and $\mathrm{O}_{2}(0,1)$ nightglow emissions during the ALOHA-93 campaign, Geophys. Res. Lett., 22, 2833-2836, 1995.

Taylor, M.J., W.R. Pendleton Jr., S. Clark, H. Takahashi, D. Gobi, and RA Goldberg Image measurements of short-period gravity waves at equatorial latitudes, J. Geophys. Res., 102, D22, 26,283-26,299, 1997.

M.J. Taylor, S.H Seo, Space Dynamics Laboratory, Utah State University, Logan, UT 84322-4145, USA (e-mail: mtaylor@ocusu.edu; Tel: 435-797-3919/3519).

T. Nakamura, T. Tsuda, Radio Atmospheric Science Center, Kyoto University, Uji, Kyoto 611, Japan.

H. Fukunishi, Y. Takahashi, Department of Geophysics, Tohoku University, Sendai, Japan.

(Received July 11, 1997; revised November 20, 1997; accepted December 29, 1997.) 